\title{
Transitivity of Heisenberg group extensions of hyperbolic systems
}

\author{
Ian Melbourne \\ Department of Mathematics \\ University of Surrey \\ Guildford, Surrey GU2 7XH, UK \\ Viorel Niţică* \\ Department of Mathematics \\ West Chester University \\ West Chester, PA 19383, USA \\ Andrei Török* \\ Department of Mathematics \\ University of Houston \\ Houston, TX 77204-3008, USA
}

May 12, 2010

\begin{abstract}
We show that among $C^{r}$ extensions $(r>0)$ of a uniformly hyperbolic dynamical system with fiber the standard real Heisenberg group $\mathcal{H}_{n}$ of dimension $2 n+1$, those that avoid an obvious obstruction to topological transitivity are generically topological transitive. Moreover, if one considers extensions with
\end{abstract}

*and Institute of Mathematics of the Romanian Academy, P.O. Box 1-764, RO-70700 Bucharest, Romania 
fiber a connected nilpotent Lie group with compact commutator subgroup (for example, $\mathcal{H}_{n} / \mathbb{Z}$ ), among those that avoid the obvious obstruction, topological transitivity is open and dense.

\section{Introduction}

This paper is part of a sequence aiming to study topological transitivity in various classes of noncompact group-extensions of hyperbolic systems. Consider a continuous transformation $f: X \rightarrow X$, a Lie group $\Gamma$, and a continuous map $\beta: X \rightarrow \Gamma$ called a cocycle. These determine a skew product, or $\Gamma$-extension,

$$
f_{\beta}: X \times \Gamma \rightarrow X \times \Gamma, \quad f_{\beta}(x, \gamma)=(f x, \gamma \beta(x))
$$

The $\Gamma$-extension $f_{\beta}$ is called topologically transitive, or simply transitive, if it has a dense orbit. The problem of interest to us is whether noncompact Lie group extensions of a hyperbolic basic set are typically topologically transitive.

Let $\left(M, d_{M}\right)$ be a smooth manifold endowed with a Riemannian metric. Let $f: M \rightarrow M$ be a smooth diffeomorphism and $X \subset M$ a compact and $f$-invariant subset of $M$. Let $D f$ be the derivative of $f$. We recall that $X$ is said to be hyperbolic if there exists a continuous $D f$-invariant splitting $E^{s} \oplus E^{u}$ of the tangent bundle $T_{X} M$ and constants $C_{1}>0,0<\lambda<1$, such that for all $n \geq 0$ and $x \in X$ we have:

$$
\begin{aligned}
\left\|\left(D f^{n}\right)_{x} v\right\| & \leq C_{1} \lambda^{n}\|v\|, \quad v \in E_{x}^{s} \\
\left\|\left(D f^{-n}\right)_{x} v\right\| & \leq C_{1} \lambda^{n}\|v\|, \quad v \in E_{x}^{u} .
\end{aligned}
$$

We say that $X$ is locally maximal if there exists an open neighborhood $U$ of $X$ such that every compact $f$-invariant set of $U$ is contained in $X$. A locally maximal hyperbolic set $X$ is a hyperbolic basic set if $f: X \rightarrow X$ is transitive and $X$ does not consist of a single periodic orbit.

Given a connected Lie group $\Gamma$ and a $C^{r}$ cocycle $\beta: X \rightarrow \Gamma, r>0$, we consider the $\Gamma$-extension $f_{\beta}: X \times \Gamma \rightarrow X \times \Gamma$ given by $f_{\beta}(x, \gamma)=(f x, \gamma \beta(x))$. For brevity, we say that the cocycle $\beta$ is transitive if the $\Gamma$-extension $f_{\beta}$ is transitive. In [6] we proposed a general conjecture about transitivity: namely that modulo obstructions appearing from the fact that the range of the cocycle is included in a maximal semigroup with non-empty interior, the set of $C^{r}$ transitive cocycles contains an open and dense subset. The conjecture is proved for various classes of Lie groups, mostly semidirect products of compact and Euclidean, in [2, 5, 6, 7, 10]. An important test case is 
presented by the special Euclidean group $\Gamma=S E(n)=S O(n) \ltimes \mathbb{R}^{n}$. It is shown in [5, 6, 7] that when $n$ is even the set of cocycles that are transitive is open and dense.

In [8] we showed that for $S E(n), n \geq 3$ odd, the transitive $C^{r}$ cocycles form a residual subset of the space of all $C^{r}$ cocycles for all $r>0$. In other words, transitivity is generic for such extensions. More generally, we considered Euclidean-type groups of the form $\Gamma=G \ltimes \mathbb{R}^{n}$ where $G$ is a compact connected Lie group. The general case of the conjecture remains unsolved for $S E(n)$ if $n \geq 3$ odd.

Recently, 9] obtained examples of groups that are compact extensions of nilpotent (not abelian) Lie groups for which transitivity is open and dense. Recall that a compact element $g$ in a Lie group is one for which the closure of the cyclic group generated by $g$ is compact. The method used in [9] borrows from [6] as it relies on the existence of an open and dense set of compact elements in $\Gamma$. This approach cannot be applied to the case of a nilpotent Lie group due to the lack of compact elements.

In this paper we study the conjecture for certain 2-step nilpotent Lie groups.

Definition 1.1 For $n \geq 1$, let $\mathcal{H}_{n}$ denote the Heisenberg group, consisting of matrices of the form

$$
(a, b, c):=\left(\begin{array}{ccc}
1 & a^{T} & c \\
0 & I_{n} & b \\
0 & 0 & 1
\end{array}\right) \in \operatorname{Mat}_{n+2}(\mathbb{R}) .
$$

where $a, b \in \mathbb{R}^{n}, c \in \mathbb{R}$ and $I_{n}$ is the $n$-dimensional identity matrix.

Remark 1.2 (a) We can identify $\mathcal{H}_{n}$ with the space $\mathbb{R}^{n} \oplus \mathbb{R}^{n} \oplus \mathbb{R}$ endowed with the multiplication

$$
(a, b, c)\left(a^{\prime}, b^{\prime}, c^{\prime}\right)=\left(a+a^{\prime}, b+b^{\prime}, c+c^{\prime}+a^{T} b^{\prime}\right) .
$$

(b) $\mathcal{H}_{1}$ is the standard 3 -dimensional Heisenberg group.

The center of $\mathcal{H}_{n}$ is $\left[\mathcal{H}_{n}, \mathcal{H}_{n}\right]=\{(0,0, c)\}=\mathbb{R}$. Denote $\widehat{\mathcal{H}}_{n}=\mathcal{H}_{n} / \mathbb{R} \cong \mathbb{R}^{2 n}$. If $\beta: X \rightarrow \mathcal{H}_{n}$ is a cocycle, denote by $\widehat{\beta}: X \rightarrow \mathbb{R}^{2 n}$ the corresponding quotient cocycle. There is an obvious obstruction to transitivity, namely that $\widehat{\beta}: X \rightarrow \mathbb{R}^{2 n}$ takes values in a half-space bounded by a hyperplane passing through the origin. To avoid repetition, we assume from now on that a half-space in a linear space is always bounded by a hyperplane passing through the origin. More generally, if $\widehat{\beta}$ is cohomologous to a cocycle with values in a half-space, then $f_{\beta}$ is not transitive ${ }^{1}$

${ }^{1}$ We recall that $\beta, \beta^{\prime}: X \rightarrow \mathbb{R}^{d}$ are cohomologous if there exists a map $P: X \rightarrow \mathbb{R}^{d}$ such that for all $x \in X, \beta^{\prime}(x)=P(f x)+\beta(x)-P(x)$. 
Remark 1.3 By [1], a Hölder $\mathbb{R}^{d}$-valued cocycle is cohomologous to one that takes values in a half-space if and only if its periodic data is in a half-space.

If $r>0$, let $\mathcal{S}^{r}\left(X, \mathcal{H}_{n}\right)$ be the set of $C^{r}$ cocycles $\beta: X \rightarrow \mathcal{H}_{n}$ for which $\widehat{\beta}$ is not cohomologous to a cocycle with values in a half-space. Our first main result is

Theorem 1.4 Assume that $X$ is a hyperbolic basic set for $f: X \rightarrow X$. Let $n \geq 1$, $r>0$. Among cocycles $\beta \in \mathcal{S}^{r}\left(X, \mathcal{H}_{n}\right)$, those that are transitive are generic.

There is a class of nilpotent groups for which we obtain stronger results, and for which the proofs are much simpler.

Let $\Gamma$ be a nilpotent Lie group, with compact commutator subgroup $[\Gamma, \Gamma]$. Note that, by [13, Lemma 4], in this case $[\Gamma, \Gamma]$ is central, hence $\Gamma$ is two-step nilpotent. For a cocycle $\beta: X \rightarrow \Gamma$, denote its image in the abelianization of $\Gamma$ by $\widehat{\beta}: X \rightarrow$ $\Gamma /[\Gamma, \Gamma]$. Since $\Gamma /[\Gamma, \Gamma]$ is an abelian Lie group, it is isomorphic to $\mathbb{R}^{a} \times \mathbb{T}^{b}$. Denote by $\widetilde{\beta}: X \rightarrow \mathbb{R}^{a}$ the image of $\widehat{\beta}$ in $\mathbb{R}^{a}$.

For $r>0$, let $\mathcal{S}^{r}(X, \Gamma)$ be the set of $C^{r}$ cocycles $\beta: X \rightarrow \Gamma$ for which $\widetilde{\beta}$ is not cohomologous to a cocycle with values in a half-space.

Theorem 1.5 Let $\Gamma$ be a nilpotent Lie group with $[\Gamma, \Gamma]$ compact, and $r>0$. Assume that $X$ is a hyperbolic basic set for $f: X \rightarrow X$. Among cocycles $\beta \in \mathcal{S}^{r}(X, \Gamma)$, those that are transitive contain an open and dense set.

One group $\Gamma$ for which the above Theorem applies is the quotient $\mathcal{H}_{n} / \mathbb{Z}$, where the central subgroup $\mathbb{Z}$ is generated by $(0,0,1)$. In this case the abelianization is $\mathbb{R}^{2 n}$. Other examples are products of such groups, and $\left(\mathcal{H}_{n} / \mathbb{Z}\right) \times \mathbb{R}^{d}$.

Remarks 1.6 1. The set $\mathcal{S}$ of cocycles considered in Theorems 1.4 and 1.5 is exactly the one to which our conjecture [6] refers: those that are not cohomologous to a cocycle with values in a maximal semigroup with non-empty interior.

2. In order to prove Theorem 1.4, it is enough to show that $\mathcal{S}^{r}\left(X, \mathcal{H}_{n}\right)$ contains a dense set of cocycle that are transitive (see for example the introduction in [8]).

3. As was the case in [8] for certain Euclidean-type groups (e.g. $S E(3)$ ), replacing generic by open and dense in Theorem 1.4 remains an unsolved problem.

4. The proof of Theorem 1.4 develops further the techniques in [8]. In addition, results from the classical theory of Diophantine approximation come into play. It is not hard to extend Theorem 1.4 to products of Heisenberg groups. We 
believe that this method works for general two-step nilpotent Lie groups (and perhaps also for nilpotent Lie groups of step higher than two) but there are several technical details that have to be clarified.

The remainder of the paper is organized as follows. In Section 2 we recall some general results from [6], in particular a general criterion for transitivity of extensions of hyperbolic systems. In Section 3 we review and extend results for $\mathbb{R}^{d}$-extensions and their periodic data. In Section 4 we prove Theorem 1.5 . In Section 5 we recall a technical result from [8]. In Section 6 we specialize to the setting of nilpotent Lie groups and prove Theorem 1.4 .

\section{Criterion for transitivity}

Let $\Gamma$ be a connected Lie group with Lie algebra $L \Gamma$. We denote by $e_{\Gamma}$ the identity element of $\Gamma$. Let Ad denote the adjoint representation of $\Gamma$ on $L \Gamma$. Let $\|\cdot\|$ be a norm on $L \Gamma$. It is known that there is a metric $d$ on $\Gamma$ with the following properties:

1. $d\left(\gamma \gamma_{1}, \gamma \gamma_{2}\right)=d\left(\gamma_{1}, \gamma_{2}\right)$;

2. $d\left(\gamma_{1} \gamma, \gamma_{2} \gamma\right) \leq\|\operatorname{Ad}(\gamma)\| d\left(\gamma_{1}, \gamma_{2}\right)$;

for any $\gamma, \gamma_{1}, \gamma_{2} \in \Gamma$.

Definition 2.1 Let $f: X \rightarrow X$ be a map and $\beta: X \rightarrow \Gamma$ a cocycle. We write $f_{\beta}^{k}(x, \gamma)=\left(f^{k} x, \gamma \beta(k, x)\right)$ where, for $k \geq 1$,

$$
\beta(k, x)=\beta(x) \beta(f x) \cdots \beta\left(f^{k-1} x\right)=\prod_{j=0}^{k-1} \beta\left(f^{j} x\right) .
$$

The meaning of the product notation in the last term above is the middle expression.

If $Q$ is a trajectory of $f$ of length $k$ (i.e. $Q=\left\{x, f(x), \ldots, f^{k-1}(x)\right\}$ for some $x$ ), then we define the height of $\beta$ over $Q$ to be $\beta(Q)=\beta(k, x)$. In particular, if $x$ is a periodic point of period $\ell$, then the height of the corresponding periodic orbit $P$ is $\beta(P)=\beta(\ell, x)$. The set of heights of $\beta$ over all periodic orbits of $f$ is referred to as the periodic data of $\beta$.

Definition 2.2 Given a cocycle $\beta: X \rightarrow \Gamma$ over $f: X \rightarrow X$, define $\mu \geq 1$ to be

$$
\mu=\max \left\{\lim _{n \rightarrow \infty} \sup _{x \in X}\|\operatorname{Ad}(\beta(n, x))\|^{1 / n}, \lim _{n \rightarrow \infty} \sup _{x \in X}\left\|\operatorname{Ad}(\beta(n, x))^{-1}\right\|^{1 / n}\right\} .
$$


We say that the cocycle $\beta$ has subexponential growth if $\mu=1$.

Remark 2.3 The subexponential growth condition is automatically satisfied for any cocycle if the group $\Gamma$ is compact, nilpotent, or a semidirect product of compact and nilpotent.

One of the key notions used in this paper was introduced in [6]:

Definition 2.4 Let $\Gamma$ be a connected Lie group, $X$ a hyperbolic basic set for $f$ : $X \rightarrow X, \beta: X \rightarrow \Gamma$ a cocycle, and $f_{\beta}: X \times \Gamma \rightarrow X \times \Gamma$ the skew-extension. Given $x \in X$, let

$$
\begin{array}{r}
\mathcal{L}_{\beta}(x)=\left\{\gamma \in \Gamma: \text { there exist } x_{k} \in X \text { and } n_{k}>0\right. \text { such that } \\
\left.x_{k} \rightarrow x \text { and } f_{\beta}^{n_{k}}\left(x_{k}, e_{\Gamma}\right) \rightarrow(x, \gamma)\right\} .
\end{array}
$$

That is, the set $\mathcal{L}_{\beta}(x)$ consists of the possible limits $\lim _{k \rightarrow \infty} \beta\left(n_{k}, x_{k}\right)$, subject to $x_{k} \rightarrow x$ and $f^{n_{k}}\left(x_{k}\right) \rightarrow x$. Note that we do not require that $n_{k} \rightarrow \infty$ or that $x_{k} \neq x$. Clearly $\mathcal{L}_{\beta}(x)$ is a closed subset of $\Gamma$.

The following theorem is a special case of [6, Lemma 3.1, Theorem 3.3].

Theorem 2.5 Assume that $X$ is a hyperbolic basic set for $f: X \rightarrow X$, that $\Gamma$ is a connected Lie group and that $\beta: X \rightarrow \Gamma$ is a Hölder cocycle that has subexponential growth. Then

(a) $\mathcal{L}_{\beta}(x)$ is a closed semigroup of $\Gamma$ for each $x \in X$.

(b) $\beta$ is a transitive cocycle if and only if there exists a point $x_{0} \in X$ such that $\mathcal{L}_{\beta}\left(x_{0}\right)=\Gamma$.

Denote by $W^{s}(x)\left(W_{l o c}^{s}(x)\right)$ and $W^{u}(x)\left(W_{l o c}^{u}(x)\right)$ the (local) stable and (local) unstable leaves of $f$ through $x$. The next lemma is a consequence of [11, Appendix A].

Lemma 2.6 Assume that $X$ is a hyperbolic basic set for $f: X \rightarrow X$, that $\Gamma$ is a connected Lie group and that $\beta: X \rightarrow \Gamma$ is an $\alpha$-Hölder cocycle that has subexponential growth. Then the $\Gamma$-extension $f_{\beta}: X \times \Gamma \rightarrow X \times \Gamma$ admits stable and unstable foliations which are $\alpha$-Hölder and invariant under right multiplication by elements of $\Gamma$. The stable and unstable leaves of $f_{\beta}$ through $\left(x, e_{\Gamma}\right) \in X \times \Gamma$ are the graphs of the functions

$$
\begin{aligned}
& \gamma_{x}^{s}: W^{s}(x) \rightarrow \Gamma, \quad \gamma_{x}^{s}(y)=\lim _{n \rightarrow \infty} \beta(n, x) \beta(n, y)^{-1}, \\
& \gamma_{x}^{u}: W^{u}(x) \rightarrow \Gamma, \quad \gamma_{x}^{u}(y)=\lim _{n \rightarrow \infty} \beta(-n, x) \beta(-n, y)^{-1} .
\end{aligned}
$$


These functions are $\alpha$-Hölder and vary continuously with the cocycle $\beta$ in the following sense: if $\beta_{k} \rightarrow \beta$ in $C^{0}$-topology and $\beta_{k}$ remains $C^{\alpha}$-bounded, then $\gamma_{k, x}^{s} \rightarrow \gamma_{x}^{s}$ on $W_{l o c}^{s}(x)$ and $\gamma_{k, x}^{u} \rightarrow \gamma_{x}^{u}$ on $W_{l o c}^{u}(x)$ in $C^{0}$-topology.

We call the values of the functions $\gamma_{x}^{s}, \gamma_{x}^{u}$ holonomies along stable/unstable leaves.

The following lemma is a special case of [6, Lemma 2.2].

Lemma 2.7 Assume that $X$ is a hyperbolic basic set for $f: X \rightarrow X$, that $\Gamma$ is a connected Lie group and that $\beta: X \rightarrow \Gamma$ is an $\alpha$-Hölder cocycle that has subexponential growth. Then there is a constant $C>0$ with the following property.

Given $\varepsilon>0$ sufficiently small and $n \geq 1$, assume that there are two trajectories $x_{k}=f^{k}\left(x_{0}\right), y_{k}=f^{k}\left(y_{0}\right)$, such that $d_{M}\left(x_{k}, y_{k}\right)<\varepsilon$ for $0 \leq k \leq n-1$. Then

$$
d\left(\beta\left(n, x_{0}\right), \beta\left(n, y_{0}\right)\right) \leq C\left(\left\|A d\left(\beta\left(n, x_{0}\right)\right)\right\|+1\right) \varepsilon^{\alpha} .
$$

\section{$3 \quad \mathbb{R}^{d}$-valued cocycles and periodic data}

In this section, we review and extend results on $\mathbb{R}^{d}$-extensions. As always, $X \subset M$ is a hyperbolic basic set for $f: M \rightarrow M$. Let $\mathcal{S}^{r}\left(X, \mathbb{R}^{d}\right)$ be the set of $C^{r}$ cocycles $\beta: X \rightarrow \mathbb{R}^{d}$ that are not cohomologous to a cocycle with values in a half-space in $\mathbb{R}^{d}$. More generally, if $K$ is a connected compact Lie group, denote by $\mathcal{S}^{r}\left(X, \mathbb{R}^{d} \times K\right)$ the $C^{r}$-cocycles $\beta: X \rightarrow \mathbb{R}^{d} \times K$ whose $\mathbb{R}^{d}$-component is not cohomologous to a cocycle with values in a half-space in $\mathbb{R}^{d}$. A necessary condition for transitivity is that $\beta \in \mathcal{S}^{r}\left(X, \mathbb{R}^{d} \times K\right)$.

First we recall a result of [10, 2, 7].

Theorem 3.1 There is an open and dense set $\mathcal{U} \subset \mathcal{S}^{r}\left(X, \mathbb{R}^{d} \times K\right)$ such that if $\beta \in \mathcal{U}$, then $\beta: X \rightarrow \mathbb{R}^{d} \times K$ is transitive.

Next, we prove that one can prescribe periodic data for Hölder $\mathbb{R}^{d}$-cocycles. This is needed in the proof of Theorem 1.4 .

Proposition 3.2 Suppose that $\beta: X \rightarrow \mathbb{R}^{d}$ is a Hölder cocycle and $U \subset X$ is an open set.

(a) Assume that $\beta$ is transitive. Then the periodic data of $\beta$ on orbits that intersect $U$ is dense in $\mathbb{R}^{d}$. 
(b) Assume that $\beta$ is stably transitive. Then, given any $\varepsilon>0$ and $a \in \mathbb{R}^{d}$, there exists a $C^{r}$ cocycle $\beta^{\prime}: X \rightarrow \mathbb{R}^{d}$ E-close to $\beta$ in the $C^{r}$-topology, such that $\beta$ and $\beta^{\prime}$ differ only on $U$ and $a$ is the periodic data of $\beta^{\prime}$ on a periodic orbit intersecting $U$.

Proof (a) Pick an open set $V \subset U$ with $\bar{V} \subset U$. Let $a \in \mathbb{R}^{d}$ and $\delta>0$. By the transitivity of $\beta$, there is a point $x_{0} \in V$ and an orbit segment $x_{0}, f\left(x_{0}\right), \ldots, f^{N}\left(x_{0}\right)$ such that $d_{M}\left(x_{0}, f^{N}\left(x_{0}\right)\right)<\delta$ and $\left\|\beta\left(N, x_{0}\right)-a\right\|<\delta$. Anosov's Closing Lemma [3] implies that there is a periodic orbit $y_{0}, f\left(y_{0}\right), \ldots, f^{N}\left(y_{0}\right)=y_{0}$ such that $d_{M}\left(f^{k}\left(x_{0}\right), f^{k}\left(y_{0}\right)\right)<$ $C \delta$ for $0 \leq k \leq N-1$, where $C$ depends only on $f$. The estimate (2.1) shows then that $\beta\left(N, y_{0}\right)$ is within $C^{\prime} \delta^{\alpha}$ of $a$, where $C^{\prime}$ depends only on $f$ and $\|a\|$. If $\delta$ is small enough then $y_{0} \in U$, and the conclusion follows.

For part (b), fix a $C^{\infty}$ bump function $g: X \rightarrow[0,1]$ supported in $U$ such that $\left.g\right|_{V} \equiv 1$ for an open set $V \subset U$. Let $M=\|g\|_{C^{r}}$. By part (a), there exists a periodic orbit $P$ intersecting $V$ with height $\beta(P)=b$ such that $\|a-b\|<\varepsilon / M$. Let $c=g(P)$ be the height of $g$ on $P$. Since $P$ intersects $V$, we have $c \geq 1$. Define $\beta^{\prime}=\beta+(a-b) g / c$. Then $\left\|\beta^{\prime}-\beta\right\|_{C^{r}} \leq\|a-b\|\|g\|_{C^{r}}<\varepsilon$, and $\beta^{\prime}(P)=\beta(P)+(a-b)=a$ as required.

\section{Open and dense transitivity (compact commu- tator subgroup)}

In this section we prove Theorem 1.5. We start with a preliminary result that is of independent interest.

Proposition 4.1 Let $\Gamma$ be a connected finite dimensional Lie group. Suppose that

(a) $N$ is a compact normal subgroup of $\Gamma$ with quotient group $H$ and natural projection $\pi: \Gamma \rightarrow H$.

(b) The only closed connected subgroup $\Gamma^{\prime} \subset \Gamma$ satisfying $\pi\left(\Gamma^{\prime}\right)=H$ is $\Gamma^{\prime}=\Gamma$.

Assume that the cocycle $\beta: X \rightarrow \Gamma$ has subexponential growth, and let $\beta_{H}: X \rightarrow H$ be the cocycle obtained after quotienting by $N$.

Then $\beta: X \rightarrow \Gamma$ is transitive if and only if $\beta_{H}: X \rightarrow H$ is transitive.

Proof We begin with the observation:

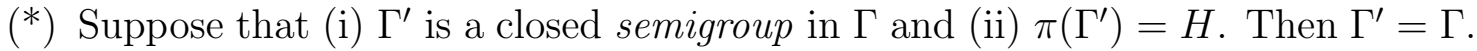


For suppose that $\gamma \in \Gamma^{\prime}$. By (ii), there exists $n \in N$ such that $\gamma^{-1} n \in \Gamma^{\prime}$. Hence $n \in \Gamma^{\prime}$. But $n$ lies in the compact group $N$, so the closed semigroup generated by $n$ is a group. In particular $n^{-1} \in \Gamma^{\prime}$ and so $\gamma^{-1} \in \Gamma^{\prime}$. Combined with (i), this shows that $\Gamma^{\prime}$ is a closed subgroup of $\Gamma$. By (ii), $\pi\left(\Gamma^{\prime}\right)=H$. By hypothesis (b), $\Gamma^{\prime}=\Gamma$ as required.

Choose $x_{0} \in X$. By Theorem 2.5(b), it suffices to show that $\mathcal{L}_{\beta}\left(x_{0}\right)=\Gamma$ if and only if $\mathcal{L}_{\beta_{H}}\left(x_{0}\right)=H$. Clearly, if $\mathcal{L}_{\beta}\left(x_{0}\right)=\Gamma$, then $\mathcal{L}_{\beta_{H}}\left(x_{0}\right)=H$. It remains to prove the converse.

So, suppose that $\mathcal{L}_{\beta_{H}}\left(x_{0}\right)=H$. We show that $\Gamma^{\prime}=\mathcal{L}_{\beta}\left(x_{0}\right)$ satisfies the hypotheses of $\left({ }^{*}\right)$, and so $\mathcal{L}_{\beta}\left(x_{0}\right)=\Gamma$ as required. Now (i) is immediate by Theorem 2.5(a). Let $\gamma \in \Gamma$. Then $\gamma N \in H$ and there exists $x_{k} \in X$ and $m_{k}>0$ such that $f^{m_{k}} x_{k} \rightarrow x_{0}$ and $\beta_{H}\left(x_{k}, m_{k}\right) \rightarrow \gamma N$. Hence $\beta\left(x_{k}, m_{k}\right)=\gamma_{k} n_{k}$ where $\gamma_{k} \rightarrow \gamma$ and $n_{k} \in N$. Since $N$ is compact, we can pass to a subsequence so that $n_{k} \rightarrow n \in N$. Hence $\gamma n \in \mathcal{L}_{\beta}\left(x_{0}\right)$. Thus (ii) is satisfied.

Remark 4.2 If $A \subset G$ is a subgroup of a nilpotent group, then $A[G, G]=G$ implies that $A=G$ (see [4, Theorem 16.2.5]).

\section{Proof of Theorem 1.5}

Since $\Gamma$ is nilpotent, cocycles $\beta: X \rightarrow \Gamma$ automatically have subexponential growth. By Remark 4.2, $\Gamma$ and its compact commutator subgroup $N=[\Gamma, \Gamma]$ satisfy the hypotheses of Proposition 4.1. (This is not so hard to check directly for $\Gamma=$ $\mathcal{H}_{n} / \mathbb{Z}$.)

Let $\beta \in \mathcal{S}^{r}(X, \Gamma)$. So the quotient cocycle $\widetilde{\beta}: X \rightarrow \mathbb{R}^{a}$ is not cohomologous to a cocycle with values in a half-space in $\mathbb{R}^{a}$, where $\Gamma /[\Gamma, \Gamma] \cong \mathbb{R}^{a} \times \mathbb{T}^{b}$. By Theorem 3.1, there is an open and dense set $\mathcal{U} \subset \mathcal{S}^{r}(X, \Gamma)$ such that if $\beta \in \mathcal{U}$, then $\widehat{\beta}: X \rightarrow \Gamma /[\Gamma, \Gamma]$ is transitive. By Proposition 4.1, $\beta: X \rightarrow \Gamma$ is also transitive.

\section{Elements of $\mathcal{L}_{\beta}$}

In this section we recall a method to obtain elements of $\mathcal{L}_{\beta}$ introduced in [8]. Throughout, $\left(M, d_{M}\right)$ is a Riemannian manifold, $X \subset M$ a hyperbolic basic set for $f: X \rightarrow X$, $\Gamma$ a connected Lie group and $\beta: X \rightarrow \Gamma$ a Hölder cocycle that has subexponential growth. 
Definition 5.1 By a periodic heteroclinic cycle we mean a cycle consisting of points $p_{1}, \ldots, p_{k}$ that are periodic for the map $f$, have disjoint trajectories, and such that $p_{j}$ is transverse heteroclinic to $p_{j+1}$ through a point $\zeta_{j} \in W^{u}\left(p_{j}\right) \cap W^{s}\left(p_{j+1}\right)$, for $j=1, \ldots, k\left(\right.$ where $\left.p_{k+1}=p_{1}\right)$.

Let $P_{1}, \ldots, P_{k}$ be the corresponding periodic orbits and denote the periods by $\ell_{1}, \ldots, \ell_{k}$. Denote by $O_{j}$ the heteroclinic trajectory from $p_{j}$ to $p_{j+1}$ (of the point $\zeta_{j}$ chosen above), and by $H_{j}$ the holonomy along this heteroclinic connection (that is, along $W^{u}\left(p_{j}\right)$ from $p_{j}$ to $\zeta_{j}$ and then along $W^{s}\left(p_{j+1}\right)$ from $\zeta_{j}$ to $\left.p_{j+1}\right)$.

Replace the heteroclinic orbit $O_{j}$ from $p_{j}$ to $p_{j+1}$ by the trajectory $Q_{j}$ of length $\ell_{j} M_{j}+\ell_{j+1} M_{j+1}$ that spends time $\ell_{j} M_{j}$ in the first half of $O_{j}$ and time $\ell_{j+1} M_{j+1}$ in the second half of $O_{j}$; that is, $Q_{j}=\left\{f^{n}\left(\zeta_{j}\right) \mid-\ell_{j} M_{j} \leq n<0\right\} \cup\left\{f^{n}\left(\zeta_{j}\right) \mid 0 \leq n<\right.$ $\left.\left.\ell_{j+1} M_{j+1}\right\}\right)$. For the trajectory connecting $p_{k}$ to $p_{k+1}$, we allow $M_{1}$ and $M_{k+1}$ to be distinct. The positive integers $M_{j}$ will be chosen later.

Consider the heights $\beta\left(P_{j}\right)$ and $\beta\left(Q_{j}\right)$ over the periodic orbits $P_{j}$ and trajectories $Q_{j}$ (see Definition 2.1).

Lemma 5.2 For $j=1, \ldots, k$, the limit

$$
\lim _{M_{j}, M_{j+1} \rightarrow \infty} \beta\left(P_{j}\right)^{-M_{j}} \beta\left(Q_{j}\right) \beta\left(P_{j+1}\right)^{-M_{j+1}}=H_{j}
$$

exists and is the product of the holonomies along the unstable and stable leaves of $O_{j}$, from $p_{j}$ to $p_{j+1}$.

Proof This follows from Lemma 2.6.

Definition 5.3 Consider a sequence of vectors $N(1), N(2) \ldots \in \mathbb{N}^{k+1}$ whose entries are positive integers. Write $N(i)=\left(M_{1}(i), \ldots, M_{k+1}(i)\right)$. The sequence is admissible if there is a constant $C_{2} \geq 1$ such that $M_{p}(i) / M_{q}(i) \leq C_{2}$ for all $p, q=1 \ldots, k+1$ and all $i \geq 1$.

If $N=\left(M_{1}, \ldots, M_{k+1}\right)$ is a sequence of vectors, we write $N \rightarrow \infty$ if $M_{p} \rightarrow \infty$ for each $p=1, \ldots, k+1$. (For an admissible sequence, this is equivalent to $M_{p} \rightarrow \infty$ for at least one value of $p$.)

The following result is [8, Theorem 3.4].

Theorem 5.4 Let $N=\left(M_{1}, \ldots, M_{k+1}\right) \in \mathbb{N}^{k+1}$. Define

$$
A(N)=\beta\left(P_{1}\right)^{M_{1}} H_{1} \beta\left(P_{2}\right)^{2 M_{2}} H_{2} \cdots \beta\left(P_{k}\right)^{2 M_{k}} H_{k} \beta\left(P_{1}\right)^{M_{k+1}} .
$$


If the limit $A=\lim _{N \rightarrow \infty} A(N)$ exists along an admissible sequence $N(1), N(2), \ldots$, then $A \in \mathcal{L}_{\beta}\left(p_{1}\right)$.

\section{Transitivity for $\Gamma=\mathcal{H}_{n}$}

In this section, we return to the Heisenberg group $\Gamma=\mathcal{H}_{n}$ and prove our main result Theorem 1.4. First, we specialize the methods of Section 5 to this situation.

As in Section 1, we denote by $\widehat{\beta}: X \rightarrow \mathbb{R}^{2 n}$ the projection of $\beta: X \rightarrow \mathcal{H}_{n}$.

By Theorem 3.1 and Proposition 3.2(b), we can assume without loss of generality that the following holds for $\beta$ and some periodic orbit $P_{1}$ :

$$
\begin{aligned}
& \beta \text { is stably transitive } \\
& \widehat{\beta}\left(P_{1}\right)=(0,0) \in \mathbb{R}^{2 n} .
\end{aligned}
$$

We will make a finite number of arbitrarily small, localized, changes to the cocycle. At each step of this process, the property (6.1) will be preserved. The main step is the following:

Lemma 6.1 Let $\beta \in \mathcal{S}^{r}\left(X, \mathcal{H}_{n}\right)$ satisfying (6.1) and $\varepsilon>0$. For any $U \subset X$ open and nonempty, and any vectors $e, f \in \mathbb{R}^{n}$ there exists $\beta^{\prime}: X \rightarrow \mathcal{H}_{n} \varepsilon$-close to $\beta$ in the $C^{r}$ topology and $h, k \in \mathbb{R}^{n}$ with the properties:

(a) for all $u, v \in \mathbb{Z}$ there exists $c \in \mathbb{R}$ such that $(h+u e, k+v f, c) \in \mathcal{L}_{\beta^{\prime}}\left(p_{1}\right)$;

(b) $\beta^{\prime}$ satisfies 6.1);

(c) the vector $(h, k) \in \mathbb{R}^{2 n}$ is the sum of holonomies for $\widehat{\beta}^{\prime}$ along a heteroclinic cycle; therefore, it can be modified by localized changes of the cocycle, while preserving properties (a) and (b) above;

(d) $\beta^{\prime}$ differs from $\beta$ only on $U$.

Proof We consider a concatenation of six periodic orbits:

$$
A(N)=\beta\left(P_{1}\right)^{M_{1}} H_{1} \beta\left(P_{2}\right)^{M_{2}} H_{2} \ldots \beta\left(P_{6}\right)^{M_{6}} H_{6} \beta\left(P_{1}\right)^{M_{7}},
$$

where $P_{1}$ is the orbit used in (6.1) and $N=\left(M_{1}, \ldots, M_{7}\right) \in \mathbb{N}^{7}$. Using the notation in (1.1), write $\beta\left(P_{i}\right)=\left(a_{i}, b_{i}, c_{i}\right), H_{i}=\left(h_{i}, k_{i}, \ell_{i}\right), i=1, \ldots, 6$. Then

$$
\beta\left(P_{i}\right)^{M_{i}} H_{i}=\left(M_{i} a_{i}+h_{i}, M_{i} b_{i}+k_{i}, M_{i} c_{i}+\ell_{i}+\frac{1}{2} M_{i}\left(M_{i}-1\right) a_{i}^{T} b_{i}+M_{i} a_{i} k_{i}\right) .
$$


Let $h=h_{1}+\cdots+h_{6}$ and $k=k_{1}+\cdots+k_{6}$. Also it is convenient to write $\left(a_{7}, b_{7}, c_{7}\right)=$ $\left(a_{1}, b_{1}, c_{1}\right)$. Using (6.2) and (6.3), we compute that $A(N)=\left(Z_{1}, Z_{2}, Z_{3}\right)$ where

$$
Z_{1}=\sum_{i=1}^{7} a_{i} M_{i}+h, \quad Z_{2}=\sum_{i=1}^{7} b_{i} M_{i}+k,
$$

and $Z_{3}$ is a quadratic polynomial in $M_{1}, \ldots, M_{7}$ with leading terms

$$
\begin{aligned}
& \frac{1}{2} \sum_{j=1}^{7}\left(a_{j}^{T} b_{j}\right) M_{j}^{2}+\sum_{1 \leq i<j \leq 7}\left(a_{i}^{T} b_{j}\right) M_{i} M_{j} \\
& \quad=\frac{1}{2}\left(\sum_{i=1}^{7} a_{i} M_{i}\right)^{T}\left(\sum_{j=1}^{7} b_{j} M_{j}\right)+\frac{1}{2} \sum_{1 \leq i<j \leq 7}\left(a_{i}^{T} b_{j}-a_{j}^{T} b_{i}\right) M_{i} M_{j} .
\end{aligned}
$$

By (6.1) and Proposition 3.2(b), we can prescribe the $\left(a_{i}, b_{i}\right)$ components of the periodic data of $P_{2}, P_{3}, P_{4}, P_{5}, P_{6}$ by making arbitrarily $C^{r}$-small perturbations of $\beta$ localized in $U$. We arrange the perturbation so that

$$
\left(a_{2}, a_{3}, a_{4}, a_{5}, a_{6}\right)=(e, 0,-8 e, 2 e, e), \quad\left(b_{2}, b_{3}, b_{4}, b_{5}, b_{6}\right)=(0, f,-f, f, 0) .
$$

We claim that with this choice of $a_{i}, b_{i} \in \mathbb{R}^{n}$, for any $u, v \in \mathbb{Z}$ there is an admissible sequence $\left(M_{1}, \ldots, M_{7}\right) \in \mathbb{N}^{7}$ that yields $A(N)=\left(Z_{1}, Z_{2}, Z_{3}\right)=\left(h+u e, k+v f, Z_{3}\right)$ with $Z_{3}$ bounded. Passing to a subsequence, we obtain that $A(N) \rightarrow(h+u e, k+v f, c)$ for some $c$.

It remains to verify the claim. First set

$$
M_{1}=M_{7}=M_{4}, M_{2}=4 M_{4}-M_{5}+u, M_{3}=M_{4}-M_{5}+v, M_{6}=4 M_{4}-M_{5} .
$$

In particular, $\sum a_{i} M_{i}=u e$ and $\sum b_{i} M_{i}=v f$, hence $Z_{1}$ and $Z_{2}$ take the required values $Z_{1}=h+u e, Z_{2}=k+v f$.

Substituting (6.6) into (6.5) gives a quadratic polynomial in $M_{4}, M_{5}$. We compute (see Remark 6.2 below) that

$$
Z_{3}=Q\left(M_{4}, M_{5}\right)=\left(e^{T} f\right)\left(4 M_{4}^{2}-8 M_{4} M_{5}+M_{5}^{2}\right)+D_{1} M_{4}+D_{2} M_{5}+E,
$$

where $D_{1}, D_{2}, E$ are real coefficients (depending on $a_{i}, b_{i}, c_{i}, h_{i}, k_{i}, \ell_{i}, u, v$ ).

The discriminant of $Q\left(M_{4}, M_{5}\right)$ is positive. Moreover the roots of $4 x^{2}-8 x+1$ are $1 \pm \frac{1}{2} \sqrt{3}$ which are positive and irrational. We take $\theta=1+\frac{1}{2} \sqrt{3}$. By Corollary A.3 
we obtain an infinite sequence $\left(M_{4}, M_{5}\right) \in \mathbb{N}^{2}$ such that $Z_{3}=Q\left(M_{4}, M_{5}\right)$ is bounded as required and moreover that

$$
M_{4} \approx \theta M_{5}
$$

Since $\theta>1$, it follows from (6.6) and (6.8) that the integers $M_{1}, \ldots, M_{7}$ are eventually positive (for $u$ and $v$ fixed) and the sequence $\left(M_{1}, \ldots, M_{7}\right)$ is admissible.

Remark 6.2 The computation required to go from $(6.5)$ and $(6.6)$ to $(6.7)$ is routine but a little tedious. It is convenient to organise the calculation as follows.

(i) The first term in (6.5) reduces to $\frac{1}{2} u v\left(e^{T} f\right)$ and hence contributes only to the constant term $E$ in (6.7).

(ii) Since $a_{1}=b_{1}=a_{7}=b_{7}=0, a_{2}=a_{6}, b_{2}=b_{6}$ and $M_{2}-M_{6}=u$, it is easily verified that the second term in (6.5) contributes to the quadratic part only $\frac{1}{2}\left\{\left(a_{3}^{T} b_{4}-a_{4}^{T} b_{3}\right) M_{3} M_{4}+\left(a_{3}^{T} b_{5}-a_{5}^{T} b_{3}\right) M_{3} M_{5}+\left(a_{4}^{T} b_{5}-a_{5}^{T} b_{4}\right) M_{4} M_{5}\right\}$ or $\left(e^{T} f\right)\left(4 M_{3} M_{4}-M_{3} M_{5}-3 M_{4} M_{5}\right)$.

(iii) Substituting $M_{3}=M_{4}-M_{5}$ gives the required result.

Remark 6.3 We note certain properties of the perturbations in Lemma 6.1. There was an initial global perturbation which ensures that the quotient cocycle $\beta: X \rightarrow$ $\mathbb{R}^{2 n}$ is stably transitive. All further perturbations preserve this property. The next perturbation is local to the vertices $p_{2}, \ldots, p_{6}$ of the heteroclinic cycle through $p_{1}$ and specifies the heights of $\widehat{\beta}$ at $P_{2}, \ldots, P_{6}$. The values of $h, k$ can then be adjusted by a further perturbation local to the heteroclinic cycle but not affecting the heights at $P_{1}, \ldots, P_{6}$.

Evidently, we may repeat the construction in Lemma 6.1 for any number of heteroclinic cycles at $p_{1}$ with localised and hence independent perturbations. In particular, for any $s \geq 1$ and any $e_{i}, f_{i} \in \mathbb{R}^{n}, i=1, \ldots, s$, there exists $\beta^{\prime}: X \rightarrow \mathcal{H}_{n}$-close to $\beta$ in the $C^{r}$ topology, and $\left(h_{i}, k_{i}\right) \in \mathbb{R}^{2 n}, i=1, \ldots, s$, given by the holonomies of each cycle, with the property:

For all $i=1, \ldots, s$, and all $u, v \in \mathbb{Z}$, there exists $c \in \mathbb{R}$ such that

$$
\left(h_{i}+u e_{i}, k_{i}+v f_{i}, c\right) \in \mathcal{L}_{\beta^{\prime}}\left(p_{1}\right) \text {. }
$$

Moreover, the central $\ell_{i}$ components of the holonomies can also be perturbed (independently of the $\left(h_{i}, k_{i}\right)$ and of the heights $\left.\beta\left(P_{i}\right)\right)$ so that the values of $c$ can be modified as necessary. 
Proof of Theorem 1.4 By Remark 6.3, we can choose $(2 n+1)$ pairs $\left(e_{i}, f_{i}\right) \in \mathbb{R}^{2 n}$ that do not lie in a half-space, and by Lemma 6.1 we obtain elements $\left(h_{i}+u_{i} e_{i}, k_{i}+\right.$ $\left.v_{i} f_{i}, c_{i}\right) \in \mathcal{L}_{\beta}\left(p_{1}\right)$ with $u_{i}, v_{i} \in \mathbb{Z}$ arbitrary. In particular, we can choose $u_{i}, v_{i}$ large and positive so that the vectors $y_{i}=\left(h_{i}+u_{i} e_{i}, k_{i}+v_{i} f_{i}\right)$ do not lie in a half-space in $\mathbb{R}^{2 n}$. We can make a further perturbation (along the heteroclinic connections) to adjust the values of $h_{i}, k_{i}$ so that the vectors $y_{1}, \ldots, y_{2 n+1}$ generate $\mathbb{R}^{2 n}$ as a closed group and hence (e.g., by [10, Lemma 5] or [7, Lemma 2.12]) as a closed semigroup.

Let $x^{*} \in \mathbb{R}^{n}-\{0\}$; for definiteness we take $x^{*}=(1,0, \ldots, 0)$. By Remark 6.3 , we can repeat Lemma 6.1 for another heteroclinic cycle; using the density of the semigroup generated by the $y_{i}$ 's, we can make an arbitrarily small perturbation to the holonomy of the new cycle to arrange that $\left(x^{*}, x^{*}, c_{1}\right) \in \mathcal{L}_{\beta}\left(p_{1}\right)$ for some $c_{1} \in \mathbb{R}$.

Repeat the above step three more times to obtain in $\mathcal{L}_{\beta}\left(p_{1}\right)$ four elements

$$
g_{1}=\left(x^{*}, x^{*}, c_{1}\right), g_{2}=\left(x^{*},-x^{*}, c_{2}\right), \quad g_{3}=\left(-x^{*}, x^{*}, c_{3}\right), g_{4}=\left(-x^{*},-x^{*}, c_{4}\right),
$$

where $c_{i} \in \mathbb{R}$ for $i=1, \ldots, 4$.

Let $c=c_{1}+c_{2}+c_{3}+c_{4}$. A calculation shows that

$$
g_{1}^{m} g_{2}^{m} g_{4}^{m} g_{3}^{m}=\left(0,0, m c-2 m^{2}\right), \quad g_{2}^{m} g_{1}^{m} g_{3}^{m} g_{4}^{m}=\left(0,0, m c+2 m^{2}\right) .
$$

For $m$ large enough, this yields elements of the form $\left(0,0, d_{1}\right),\left(0,0, d_{2}\right)$ with $d_{1}<0$, $d_{2}>0$. If necessary, we can perturb $c_{1}$ (say) so that $d_{1}$ and $d_{2}$ are incommensurate. Since $\mathcal{L}_{\beta}\left(p_{1}\right)$ is a closed semigroup, we deduce that $\mathcal{L}_{\beta}\left(p_{1}\right)$ contains the center $\mathbb{R}$.

Next we return to the elements $\left(y_{1}, t_{1}\right), \ldots,\left(y_{2 n+1}, t_{2 n+1}\right)$ where the vectors $y_{i}=$ $\left(h_{i}+u_{i} e_{i}, k_{i}+v_{i} f_{i}\right) \in \mathbb{R}^{2 n}$ do not lie in a half-space and generate (as a semigroup) a dense subset of $\mathbb{R}^{2 n}$. Since $\mathcal{L}_{\beta}\left(p_{1}\right)$ is a closed semigroup containing $\{0\} \times \mathbb{R}$, it follows that $\mathbb{R}^{2 n} \times\{0\} \subset \mathcal{L}_{\beta}\left(p_{1}\right)$ completing the proof.

\section{A Bounds for indefinite binary quadratic polyno- mials}

In this appendix, we recall classical results of Chebyshev and Minkowski that give bounds for indefinite binary quadratic polynomials along sequences of positive integers. A proof of the next theorem, due to Chebyshev, can be found in [12] (only a few steps of [12] are needed to prove (A.1) with 2 replaced by some bounded constant; that is sufficient for our purposes). Corollary A.2 is a weaker form of a result that Minkowski proved in 1901. 
Theorem A.1 (Chebyshev, 1866) Let $\theta$ be irrational and $\omega$ a real number. Then there exist an infinite number of integer pairs $(x, y), y>0$, for which the quantity $|x-\theta y-\omega|$ is made arbitrarily small while

$$
|x-\theta y-\omega|<\frac{2}{y}
$$

Corollary A.2 Let $\theta>0$ be irrational and $\omega$ a real number. Then for any real numbers $B_{1}, B_{2}, B_{3}$, there exist constants $\varepsilon \in(0, \theta)$ and $M=M\left(B_{1}, B_{2}, \theta\right)>0$, and an infinite set of distinct pairs of positive integers $(x, y)$ that satisfies

$$
\theta-\varepsilon<\frac{x}{y}<\theta+\varepsilon
$$

and

$$
\left|(x-\theta y-\omega)\left(B_{1} x+B_{2} y+B_{3}\right)\right|<M .
$$

Proof Pick a sequence of integers $(x, y)$ given by Theorem A.1. As $\theta>0$, we can assume that $x$ and $y$ have the same signs, and thus are positive.

Consider now A.1 and divide both sides by $y$. One has

$$
\left|\frac{x}{y}-\theta-\frac{\omega}{y}\right|<\frac{2}{y^{2}}
$$

which implies the existence of $\varepsilon$ and A.2.

To prove A.3, use A.1, A.2 and take $y$ sufficiently large so that

$$
\begin{gathered}
\left|(x-\theta y-\omega)\left(B_{1} x+B_{2} y+B_{3}\right)\right| \leq 2\left|B_{1} \cdot \frac{x}{y}+B_{2}+\frac{B_{3}}{y}\right| \\
\leq 2\left|B_{1}\right|(\theta+\varepsilon)+2\left|B_{2}\right|+1=M .
\end{gathered}
$$

Corollary A.3 Let $Q(x, y)=A x^{2}+B x y+C y^{2}+D x+E y+F$ be a quadratic polynomial with positive discriminant $B^{2}-4 A C>0$. Suppose that there exists $\theta>0$ irrational such that $A \theta^{2}+B \theta+C=0$. Then there exist constants $\varepsilon \in(0, \theta), M>0$ and an infinite set of positive integers $(x, y)$ such that

$$
\theta-\varepsilon<\frac{x}{y}<\theta+\varepsilon \quad \text { and } \quad|Q(x, y)|<M .
$$


Proof Since $B^{2}-4 A C \neq 0$, we can write $Q(x, y)=A \tilde{x}^{2}+B \tilde{x} \tilde{y}+C \tilde{y}^{2}+\tilde{F}$ where $\tilde{x}=x+a, \tilde{y}=y+b$ and $a, b, \tilde{F} \in \mathbb{R}$. Since $B^{2}-4 A C>0$, there are real roots $\theta_{1}, \theta_{2}$ such that $A \tilde{x}^{2}+B \tilde{x} \tilde{y}+C \tilde{y}^{2}=A\left(\tilde{x}-\theta_{1} \tilde{y}\right)\left(\tilde{x}-\theta_{2} \tilde{y}\right)$. Hence there exist $\omega_{1}, \omega_{2} \in \mathbb{R}$ such that

$$
Q(x, y)=A\left(x-\theta_{1} y-\omega_{1}\right)\left(x-\theta_{2} y-\omega_{2}\right)+\tilde{F} .
$$

At least one of the roots, say $\theta_{1}$, is irrational and positive, and we set $\theta=\theta_{1}$. The result follows from Corollary A.2.

Acknowledgments: This research was supported in part by EPSRC Grant EP/F031807/1 (IM) and by NSF Grant DMS-0600927 (AT).

\section{References}

[1] T. Bousch. La condition de Walter. Ann. Sci. Ecole Norm. Sup. 34 (2001) 287311.

[2] M. Field, I. Melbourne, A. Török. Stable ergodicity for smooth compact Lie group extensions of hyperbolic basic sets. Ergod. Th. \& Dynam. Sys. 25 (2005) 517-551.

[3] A. Katok, B. Hasselblatt, Introduction to the modern theory of dynamical systems, Cambridge University Press, 1995.

[4] M. I. Kargapolov, Ju. I Merzljakov, Fundamentals of the theory of groups, Graduate Texts in Mathematics, 62, Springer-Verlag, 1979.

[5] I. Melbourne, M. Nicol. Stable transitivity of Euclidean group extensions. Ergod. Th. \& Dynam. Sys. 23 (2003) 611-619.

[6] I. Melbourne, V. Niţică, A. Török. Stable transitivity of certain noncompact extensions of hyperbolic systems. Annales Henri Poincaré 6 (2005) 725-746.

[7] I. Melbourne, V. Niţică, A. Török. A note about stable transitivity of noncompact extensions of hyperbolic systems. Discrete \& Contin. Dynam. Systems 14 (2006) 355-363.

[8] I. Melbourne, V. Niţică, A. Török. Transitivity of Euclidean-type extensions of hyperbolic systems. Ergod. Th. \& Dynam. Sys. 29 (2009) 1585-1602. 
[9] V. Niţică. Stably transitivity for extensions of hyperbolic systems by semidirect products of compact and nilpotent Lie groups. submitted to Discrete and Continuous Dynamical Systems (2010).

[10] V. Niţică, M. Pollicott. Transitivity of Euclidean extensions of Anosov diffeomorphisms. Ergod. Th. \& Dynam. Sys. 25 (2005) 257-269.

[11] V. Niţică, A. Török. An open and dense set of stably ergodic diffeomorphisms in a neighborhood of a non-ergodic one. Topology 40 (2001) 259-278.

[12] R. Q. Seale. A new proof of Minkowski's theorem on the product of two linear forms. Bull. Amer. Math. Soc. 41 (1935) 419-426.

[13] J. Winkelmann. Generic subgroups of Lie groups. Topology 41 (2002) 163-181. 